\title{
Etude quantitative de populations de Phyllocnistis suffusella $Z$. (Lepid. Lithocolletidae) en Belgique
}

\author{
L. NEF \\ Centre de Recherches pour la Lutte intégrée contre les Insectes forestiers \\ (Université Catholique de Louvain-AGRO/EFOR). Place Croix du Sud, 2, B.P. 4, \\ B-1348 Louvain-la-Neuve (")
}

\begin{abstract}
Summary
Quantitative study of populations of Phyllocnistis suffusella (Lepid. Lithocolletidae) in Belgium
\end{abstract}

This paper concerns the absolute and the relative frequencies of Ph. suffusella, the most abundant leaf miner of the poplar in Belgium and, probably, in W.-Europe. For this work, we retained the results of 7 years in 7 populetums and on 12 clones belonging to 3 poplar groups; the trees are between 2 and 7 year old, and 5 to $15 \mathrm{~m}$ high.

The mean value is 3.8 attacked leaves per meter of twig (tabl. 2); this value shows moderate variations in the various populetums. Ph. suffusella is a common and regular insect on poplar trees; its population appears to be quantitatively regulated by accurate mechanisms.

The population densities vary considerably and significantly according to station and to genetic factors. Conversely, time (and thus climate or prey-predator relationships) has only a moderate and not significant effect on those densities. The extreme observations, pooling the effects of station, year and clone, were $0.1 \mathrm{larva} / \mathrm{m}$ for the clone Unal at Borgloon in 1978 , and $11.3 \mathrm{l} / \mathrm{m}$ for the Primo at Rotem in 1981.

On the average, 25 p. 100 of the poplar leaves are attacked, the maximum observed attaining $71 \mathrm{p}, 100):$ Ph. suffusella is therefore capable of influencing markedly the poplar trees and their growth. The above mentioned mean value differs little. but significantly from clone to clone: the extreme values are 36.6 p. 100 for Robusta and 19.0 p. 100 for Beaupré.

Key words: Phyllocnistis suffusella, poplar, Populus, leaf miner, population density.

\section{Résumé}

Le but du travail est d'exprimer les fréquences absolues et relatives de Phyllocnistis suffusella $Z$. , la mineuse la plus abondante sur les feuilles de peuplier en Belgique. La présente étude porte sur 7 années, 7 populetums, et 12 clones appartenant à 3 groupes de Populus, lâge des arbres variant entre 2 et 7 ans, et leur hauteur entre 5 et $15 \mathrm{~m}$

$\left(^{*}\right)$ Recherches subsidićes par l'Institut pour l'Encouragement de la Recherche Scientifique dans l'Industrie et l'Agriculture (I.R.S.I.A.). 
En moyennc, il y avait 3.8 feuilles attaquées par mètre de branche, les valeurs individuelles s'écartant assez peu de cette moyenne. Ph. suffusella est un insecte commun et régulier sur peuplier, dont la population doit être réglée quantitativement par des mécanismes précis.

Les facteurs stationnels et génétiques ont unc influence significative sur les densités; au contraire, le facteur temps, et donc les variations climatiques ou les relations proic-prédateur, n'ont qu'un effet modéré.

En moyenne, 25 p. 100 des feuilles des peupliers sont attaquées. la valeur la plus élevée observée étant de 71 p. 100 ; Ph. suffusella peut donc avoir une incidence réelle sur le peuplier et sa croissance. Cette moyenne diffère significativement mais modérément d'un clone à l'autre : les extrémes sont $36,6 \mathrm{p}$. 100 pour le Robusta, et $19,0 \mathrm{p}$. 100 pour le Beaupré.

Mots clés : Phyllocnistis suffusella, peuplier, Populus, mineuse, densité de population.

\section{Introduction et but}

Depuis plusieurs années, la station de Populiculture de Grammont a été particulièrement active dans la création de nouveaux clones de peuplier (STEENACKERS, 1982). Ces clones ont été étudiés à de nombreux points de vue, surtout par les membres de la C.N.B.P. (Commission Nationale Belge de Populiculture), et entre autres quant à leur sensibilité aux attaques d'insectes (NEF, 1985/A, 1985/B). Pour ces études, nous avions mis au point une méthode d'estimation de la "Sensibilité relative " (NEF, 1983), exprimant avec quelle fréquence chaque clone est plus (ou moins) attaqué que les autres par une espèce donnée d'insecte. Mais cette expression relative ne donne aucune indication sur les densités absolues de population des déprédateurs. Le but du présent travail est de fournir une telle information sur Phyllocnistis suffusella Z., la mineuse la plus abondante, et de formuler certaines conclusions à propos de cette densité et de ses variations. D'autres recherches ayant prouvé que $P h$. suffusella influence chimiquement et morphologiquement les feuilles attaquées (Bouyaïche, 1987 ; NEF, 1987), il nous a semblé indispensable d'avoir une information précise sur la fréquence de cet insecte, ce qui permettra ultérieurement d'estimer son impact sur la croissance de l'arbre.

\section{Matériel et méthodes}

\subsection{L'insecte}

En Belgique, Ph. suffusella a deux générations par an. La chenille vit comme mineuse sous l'épiderme des feuilles du peuplier, la chrysalide se formant le plus souvent dans une chambrette constituée, à l'extrémité de la mine, par un repli du bord de la feuille. La galerie est peu visible, conservant la couleur de la feuille, mais prenant un aspect brillant, rappelant celui d'une trace de limace. Plus de détails sur cet insecte et son écologie peuvent être trouvés par exemple chez ARru (1966) ou chez NeF (1982).

\subsection{Les arbres-hôtes et l'échantillonnage}

Notre étude porte sur les plus représentatifs des populetums de la C.N.B.P. que nous avons étudiés de 1977 à 1982, ainsi que sur celui de Louvain-la-Neuve (BouYaïche, 1987). 
L'échantillonnage a porté sur 4 répétitions par clone dans chaque populetum, c'està-dire sur 4 arbres, le dispositif expérimental étant du type «one tree plot». Sur chaque arbre, ont été prélevées 8 extrémités de branches de $1 \mathrm{~m}$, réparties sur deux niveaux différents : l'un à la base, l'autre vers le milieu du houppier. Sur ces branches, on a dénombré toutes les feuilles présentes, ainsi que celles attaquées par divers insectes dont $P h$. suffusella. Pour ce dernier, on peut considérer, avec une bonne approximation, qu'il y a autant de chenilles que de feuilles attaquées; en effet, Bouyaïche (com. verb.) a constaté que moins de 5 p. 100 des feuilles attaquées portaient deux galeries larvaires.

Les clones étudiés croissant de quelque $2 \mathrm{~m}$ par an, les peupliers de la pépinière de Rotem avaient environ $4 \mathrm{~m}$, ceux des divers populetums oscillaient, d'après l'âge, le clone et la station, entre 5 et $15 \mathrm{~m}$.

Les échantillonnages ayant eu lieu vers la fin de la saison, les résultats totalisent toujours les deux générations de l'année. Les clones retenus pour la présente étude, avec leurs abréviations respectives, sont cités dans le tableau 1.

TABleaU 1

Clones étudiés. / Studied clones.

\begin{tabular}{|c|c|c|c|}
\hline Groupe: & $\begin{array}{l}\text { Populus deltoides } \times \text { nigra } \\
(\mathrm{DN})\end{array}$ & $\begin{array}{l}\text { P. trichocarpa } \\
\text { (T) }\end{array}$ & $\begin{array}{l}\text { P. trichocarpa } \times \text { deltoides } \\
\text { (TD) }\end{array}$ \\
\hline Clones: & $\begin{array}{ll}\text { Robusta } & \text { (RO) } \\
\text { Gaver } & \text { (GA) } \\
\text { Gibeca } & \text { (GI) } \\
\text { Primo } & \text { (PR) } \\
\text { Ogy } & (\mathrm{OG}) \\
\text { Ghoy } & (\mathrm{GH})\end{array}$ & $\begin{array}{ll}\text { Columbia River } & \text { (CR) } \\
\text { Fritzy Pauley } & \text { (FP) } \\
\text { Trichobel } & \text { (TR) }\end{array}$ & $\begin{array}{l}\text { Unal } \quad(\mathrm{UN}) \\
\text { Beaupré }(\mathrm{BE}) \\
\text { Boelaere } \\
(\mathrm{BO})\end{array}$ \\
\hline
\end{tabular}

\section{Résultats}

Le nombre d'insectes par unité de longueur de branche informe sur la densité de population; c'est ce que nous avons considéré dans un premier temps (3.1.).

D'autre part, en vue d'estimer l'influence de l'insecte sur l'arbre-hôte, il importe de connaitre la proportion de feuilles attaquées: cette expression sera donc également calculée et interprétée (3.2.).

\subsection{Paramètre de la densité de population}

Les résultats directs des comptages sont synthétisés au tableau 2 qui donne:

- les populetums étudiés ;

- lannée de la plantation ;

- la ou les années d'étude et l'âge des arbres au moment de l'étude (nombre de saisons de croissance calculé à partir du bouturage) ; 


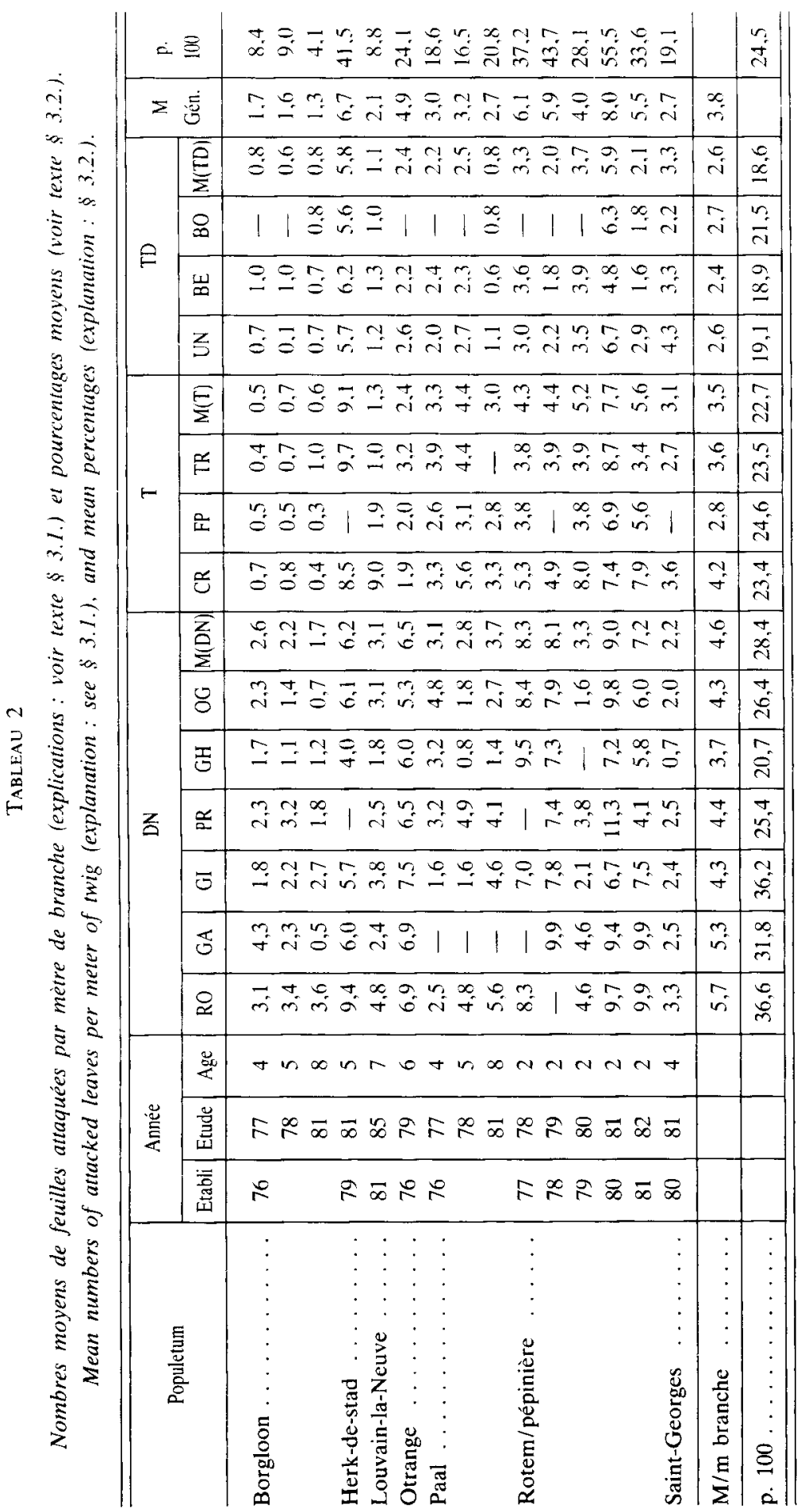


- pour chaque clone, le nombre moyen de feuilles attaquées par mètre de branche, calculé à partir des $32 \mathrm{~m}$ échantillonnés, sauf pour Louvain-la-Neuve où l'échantillonnage n'a porté que sur $20 \mathrm{~m}$; abréviations pour les clones : voir tableau 1;

- dans les lignes, les moyennes par groupes (M) ou pour l'ensemble d'un champ d'expérience (M Gén.) ;

- dans les colonnes, les moyennes par clone ( $\mathrm{M} / \mathrm{m}$ branche).

\section{Précisons que :}

- par exemple, des arbres plantés en 1976 et étudiés en 1977 ont deux années de croissance en populetum puisque la plantation a eu lieu au printemps 1976 et l'étude en automne 1977 ;

- pour la pépinière de Rotem, l'étude a toujours porté sur des pieds de deux ans, bouturés l'année précédente, et qui ont été ensuite transférés vers un populetum au cours de l'hiver suivant. D’une année à l'autre, c'étaient donc toujours des arbres différents mais de même âge qui ont été étudiés. Au contraire, dans les populetums, les études successives portaient sur les mêmes arbres d'âge croissant.

\subsection{Proportion des feuilles attaquées}

A partir du total des feuilles attaquées et du nombre de feuilles sur les branches échantillonnées pour chaque case du tableau 2, nous avons calculé les pourcentages de feuilles attaquées. L'essentiel des résultats, à savoir les moyennes par populetum/année, et par clone, figurent sous le sigle p. 100 dans le même tableau 2.

Ces nombres de feuilles varient sensiblement d'après les clones, mais aussi d'après les populetums et d’après les années comme le montrent les exemples du tableau 3.

\section{TABIEAU 3}

Exemples de nombres moyens de feuilles par $m$ de branches (pour les groupes: movennes des divers clones).

Examples of mean numbers of leaves per meter of twig (for the groups: means of the various clones).

\begin{tabular}{|c|c|c|c|c|c|c|c|}
\hline \multirow{2}{*}{ Populetum } & \multirow{2}{*}{$\begin{array}{l}\text { Année } \\
\text { d'étude }\end{array}$} & \multicolumn{3}{|c|}{ Clones } & \multicolumn{3}{|c|}{ Groupes } \\
\hline & & $\mathrm{RO}$ & $\mathrm{CR}$ & UN & DN & $\mathbf{T}$ & TD \\
\hline Borgloon & $\begin{array}{l}77 \\
78 \\
81\end{array}$ & $\begin{array}{l}20 \\
27 \\
47\end{array}$ & $\begin{array}{l}21 \\
16 \\
34\end{array}$ & $\begin{array}{l}17 \\
14 \\
26\end{array}$ & $\begin{array}{l}21 \\
17 \\
29\end{array}$ & $\begin{array}{l}19 \\
15 \\
30\end{array}$ & $\begin{array}{l}17 \\
14 \\
26\end{array}$ \\
\hline Paal & $\begin{array}{l}77 \\
78 \\
81\end{array}$ & $\begin{array}{l}12 \\
17 \\
12\end{array}$ & $\begin{array}{l}18 \\
21 \\
16\end{array}$ & $\begin{array}{l}12 \\
19 \\
14\end{array}$ & $\begin{array}{l}16 \\
19 \\
12\end{array}$ & $\begin{array}{l}17 \\
20 \\
15\end{array}$ & $\begin{array}{l}13 \\
20 \\
14\end{array}$ \\
\hline
\end{tabular}




\subsection{Traitements statistiques (1)}

Un matériel plus large mais plus irrégulier, étudié précédemment (NEF, 1982) avait montré que :

- il y a une concordance hautement significative entre les rangs que les groupes de clones occupent dans les différents populetums: il y a donc un effet groupe significatif $(\mathrm{P}<1$ p. 100 , test de Kendall) ;

- comparés deux à deux, les clones des groupes T et TD ne diffèrent pas ou guère; ceux du groupe DN, plus diversifiés dans leur sensibilité à l'insecte, peuvent différer parfois très significativement $(\mathrm{P}<1$ p. 100 , tests de Wilcoxon ou de Walsh).

Les nombres de feuilles attaquées ont d'abord fait l'objet d'analyses de variances portant sur les rangs, ceci afin de minimiser les effets des distributions non normales des données:

-- les différences entre groupes sont confirmées $(P<1$ p. 100) ;

- l'effet populetum est très hautement significatif $(\mathbf{P}<0,1$ p. 100);

- à Borgloon et Paal, l'effet âge (mêmes arbres d'âge croissant) est négligeable $(\mathrm{P}=35$ p. 100); aucune interaction n'est significative : âge $\times$ groupe $(P=46$ p. 100), âge $\times$ populetum $(\mathrm{P}=78$ p. 100), âge $\times$ groupe $\times$ populetum $(\mathrm{P}=15$ p. 100$)$;

- à Rotem (arbres différents, âge constant), l'effet année est très hautement significatif $(P=0,01$ p. 100$)$; après élimination de 1980 (voir $\$ 4.2.), P=2$ p. 100.

Pour tester l'effet clone, il a fallu recourir au test de Kendall; à l'intérieur du groupe $D N$, la concordance est très significative $(P<1$ p. 100).

Le même test de Kendall a été préféré pour étudier les pourcentages. II ressort que :

- l'effet groupe de clones n'est pas du tout significatif ;

- l'effet clone, tous groupes confondus, est hautement significatif $(\mathrm{P}<1$ p. 100) ;

- l'effet populetum l'est encore plus ( $\mathrm{P}<0,1$ p. 100) ; à Paal.

- l'effet temps est très significatif à Borgloon et à Rotem $(\mathrm{P}<1$ p. 100) mais pas

Avant de passer à l'interprétation des résultats, rappelons clairement qu'un facteur peut fort bien engendrer des différences statistiquement très significatives, mais de faible amplitude en valeur absolue. Dans une optique d'entomologie appliquée, il y aurait alors lieu de conclure que ce facteur est plutôt négligeable. L'inverse peut également se présenter.

\section{Discussion}

\subsection{Occurrence générale}

Pour l'ensemble des observations reprises au tableau 2, il y avait en moyenne 3,8 feuilles attaquées par mètre de branche (et donc, à très peu de chose près, le même

(1) Surtout pour les diverses analyses de variance, Monsicur P. Dutilleul de l'unité BIOM de IU.C.L. nous a aidés avec la pius aimable efficacité. 
nombre de larves : voir $\$ 2.2$.). Cette moyenne varie modérément d'un populetum à l'autre, les extrêmes étant de 1,3 dans le moins attaqué (Borgloon, 1981) et 8,0 à Rotem, en 1981. Ces résultats, couvrant plusieurs années et diverses situations géographiques, révèlent que $P h$. suffusella est un insecte commun et régulier dans les peupleraies.

\subsection{Variation des densités de population}

La modicité de l'écart entre les valeurs extrêmes citées au paragraphe précédent est un premier élément suggérant que les populations de $P h$. suffusella sont régularisées par des mécanismes assez précis et constants. Cette hypothèse est renforcée par la grande stabilité quantitative observée d'une année à l'autre dans les populetums échantillonnés à plusieurs reprises où les écarts entre les extrêmes sont toujours inférieurs à 50 p. 100.

Ainsi (tabl. 2), les moyennes annuelles à Borgloon n'oscillent qu'entre 1,3 et 1,7 sur une période de 4 ans, à Paal entre 2,7 et 3,2 et à Rotem entre 5,5 et 8,0 si l'on exclut les chiffres de 1980 où l'échantillonnage a été perturbé par les particularités météorologiques. Or, dans ces divers populetums, la situation diffère. Dans la pépinière de Rotem, la rotation annuelle des peupliers, déplaçant l'étude chaque année vers de nouveaux arbres, n'a pas empêché la population de n'osciller qu'entre d'étroites limites. La même constatation vaut pour les autres populetums où les arbres ont crû de manière appréciable durant la période considérée : on doit donc admettre qu'ici la population totale d'insectes a suivi une évolution qui a maintenu constante son abondance par unité d'espace de son habitat. En conclusion, dans tous ces cas, il y a une étonnante stabilité de la densité de population malgré la variation temporelle des arbres-hôtes.

Les différences spatiales sont plus importantes, les extrêmes des moyennes par populetum étant dans le rapport de 1 à 4,3. Ces différences ont une première explication: elles sont liées aux conditions édaphiques, comme nous l'avons montré précédemment (NEF, 1982).

Les valeurs individuelles par clone peuvent évidemment varier plus que les moyennes, mais l'écart maximum (Borgloon, clone GA), avec un rapport de 1 à 9 , est l'exception et en tout cas il est beaucoup inférieur à ces variations impressionnantes que présentent la plupart des insectes phytophages, et pas uniquement les espèces «à pullulation " où les rapports peuvent être de 1 à $10^{\circ}$ ou plus encore.

Le tableau 2 montre clairement l'effet des facteurs génétiques (moyennes par groupe ou par clone) sur les variations de densité, ces résultats quantifiant ceux obtenus précédemment par les «Sensibilités Relatives» (NEF, 1983 et 1985/B). Soulignons quelques valeurs typiques : $\mathrm{T} \times \mathrm{D}$

- les moyennes par groupe : 4,6 pour les $\mathrm{D} \times \mathrm{N}, 3,7$ pour les $\mathrm{T}$ et 2,6 pour les

- les extrêmes des moyennes par clone, avec 5,6 larves par mètre de branche pour Robusta et 2,4 pour Beaupré ;

- et enfin, les extrêmes par observation avec 0,1 larve $/ \mathrm{m}$ pour le clone Unal à Borgloon en 1978, et 11,3 larves/m pour le Primo à Rotem en 1981, ce qui représente une population très dense. Le rapport entre ces deux dernières populations est de 1 à 90 (calculé sur les valeurs brutes), mais il résulte de l'action combinée de tous les facteurs envisagés : année, station, clone. 
En conclusion, il apparaît que le facteur temps n'a qu'un effet très modéré sur les variations quantitatives, ce qui fait supposer que les facteurs climatiques et que les relations de type prédateurs-proies ne jouent pas de rôle prépondérant dans les régulations de densité. Par contre, les facteurs stationnels et surtout génétiques semblent les principaux déterminants du niveau général des populations de $P h$. suffusella. Le chimisme de la feuille-hôte constitue le lien le plus probable entre ces facteurs et l'in'secte ; d'autres résultats ayant démontré l'existence de relations entre $P h$. suffusella et les polyphénols ou les éléments minéraux des feuilles de peuplier (Bouyaïche \& NEF, 1987 ; NEF, 1985/A et 1987), nous croyons pouvoir considérer que la chaîne causale suivante joue un rôle majeur dans la détermination des densités de population de Ph. suffusella:

\begin{tabular}{|c|c|c|c|c|c|c|}
\hline $\begin{array}{c}\text { Facteurs } \\
\text { stationnels } \\
\text { et génétiques }\end{array}$ & $\rightarrow$ & $\begin{array}{l}\text { Chimisme } \\
\text { des feuilles } \\
\text { du peuplier }\end{array}$ & $\rightarrow$ & $\begin{array}{c}\text { Survie et } \\
\text { développement } \\
\text { larvaire }\end{array}$ & $\rightarrow$ & $\begin{array}{c}\text { Densité } \\
\text { de } \\
\text { population }\end{array}$ \\
\hline
\end{tabular}

\subsection{Paramètre de l'importance du $\mathrm{Ph}$. suffusella pour l'arbre-hôte}

Le pourcentage de feuilles attaquées constitue un paramètre de l'importance de cet insecte en tant que déprédateur du peuplier. La moyenne générale se situant vers 25 p. 100 , et si ce résultat est généralisable à l'ouest de l'Europe, on doit considérer que $P h$. suffusella est un hôte régulier du peuplier. Mais il peut parfois attaquer de grandes proportions de feuilles, le maximum observé étant de 71 p. 100 (Rotem, clone GA, 1981). D'autres recherches ont montré que la surface des feuilles attaquées est inférieure de quelque 25 p. 100 à celle des feuilles indemnes, que les teneurs en éléments minéraux et en polyphénols y sont modifiées très sensiblement et que les galeries larvaires peuvent être envahies par des micro-organismes (BouYaïche, 1987 ; NEF, 1987) ; cette mineuse perturbe donc certainement la croissance des peupliers, et on peut s'étonner du peu d'intérêt qui lui est porté : Ph. suffusella n'est même pas cité dans SCHWENKE (1978).

Tout comme les densités, les pourcentages moyens varient d'après les facteurs étudiés ; ils sont très variables pour les populetums/années (extrêmes: 55 p. 100 à Rotem en 1981, et 4 p. 100 à Borgloon en 1981) mais le sont nettement moins en fonction des clones (37 p. 100 pour le Robusta, 19 p. 100 pour le Beaupré). Statistiquement, ce dernier facteur a un effet très significatif mais, en valeur absolue, l'impact physiologique et économique de cet insecte est assez voisin pour les divers clones considérés.

Les fréquences des pourcentages calculés pour chaque case du tableau 2 (populetum $\times$ clone $\times$ année, voir $\S 3.2$.) se répartissent comme suit :

\begin{tabular}{c|c|c|c}
\hline \hline p. 100 & $\begin{array}{c}\text { Fréquence } \\
\text { d'observations }\end{array}$ & p. 10) & $\begin{array}{c}\text { Fréquence } \\
\text { d'observations }\end{array}$ \\
\hline $0-10$ & 39 & $40,1-50$ & 12 \\
$10,1-20$ & 41 & $50,1-60$ & 14 \\
$20,1-30$ & 26 & $60,1-70$ & 4 \\
$30.1-40$ & 23 & $70,1-80$ & 1 \\
\hline
\end{tabular}


Cette distribution met en évidence, par une autre approche, la « tendance centripète ", donc la stabilité spatio-temporelle, des densités de population de $P h$. suffusella.

\subsection{Conclusions annexes}

A. Nos résultats ne concernent que les jeunes peupliers; dans les limites du matériel étudié, ils ne suggèrent pas d'évolution de la population en fonction de l'âge de l'arbre, mais nous n'oserions pas en inférer que cet insecte est encore aussi nombreux sur les peupliers à maturité. Ce point mériterait vérification.

B. D'autre part, dans les peupleraies mono- ou oligoclonales, les populations pourraient différer de celles observées dans nos populetums très mélangés; il n’y a cependant aucune raison de craindre que ces différences puissent être importantes.

C. Tant envers $P h$. suffusella qu'envers plusieurs autres phytophages du peuplier $\left(N_{E F}, 1985 / B\right)$, le Robusta s'avère le plus sensible : cet insecte pourrait donc avoir surmonté les mécanismes de défense de ce clone, vieux aujourd'hui d'environ 150 ans. Si cette hypothèse devait se vérifier, ceci signifierait qu'il faut rester prudent quant à la durée de la protection assurée par la sélection génétique.

\section{Reçu le $I^{\text {er }}$ septembre 1987. Accepté le 8 février 1988.}

\section{Références bibliographiques}

Arru G., 1966. I piu importanti Insetti minatori delle foglie di Pioppo nell'Italia settentrionale Boll. Zool. agr. Bachic., II/8, 41-74.

Bouynïche M., 1987. Interactions chimiques et biologiques entre Phyllocnistis suffusella Z, et divers clones de peupliers. Mémoire, I.S.N.A.P., 106 p. U.C.L., Louvain-la-Neuve.

Bouyaïche M., Nef L., 1987. Relations entre les attaques de Phyllocnistis suffusella, la croissance et la composition minérale des feuilles de peuplier. Meded. Fac. Landbouww. Rijksun. Gent, 52, 3/A, 259-266.

NEF L., 1982. Influence des facteurs génétiques et environnementaux sur la résistance des peupliers aux attaques de Phyllocnistis suffusella. Meded. Fac. Landbouww. Rijksun. Gent, 47 (2), 597-606.

NEF L., 1983. Estimation non paramétrique de la sensibilité des plantes envers les déprédateurs phytophages. Biom. Praxim., 23, 73-82.

NeF L., 1985/A. Relations entre certaines caractéristiques des peupliers et l'abondance d'insectes phytophages. Z. ang. Ent., 99, 160-170.

NEF L., 1985/B. De populierinsekten in Belgie in de periode 1979-1983, Voorkormen, Onderzoek. Bull. Soc. R. For. B., 92 (4), 153-162.

NEF L., 1988. Reciprocal relationship between Phyllocnistis suffusella and poplar leaves. Proc. W.P. I.U.F.R.O. on Plant Resist. Mechan. to Insects, Orléans, 1986.

SChwenke W., 1978. Die Forstschädlinge Europas, Bd. 3, 467 p., P. Parey, Hamburg-Berlin.

StEenaCkers, 1982. Le travail d'amélioration du peuplier en Belgique. Forêt Entr./Bull. Vulg. For., $82(2), 4-9$. 